\title{
ON ALMOST KENMOTSU MANIFOLDS WITH NULLITY DISTRIBUTIONS
}

\author{
UDAY CHAND DE, JAE-BOK JUN AND KRISHANU MANDAL
}

\begin{abstract}
The object of this paper is to characterize the curvature conditions $R \cdot P=0$ and $P \cdot S=0$ with its characteristic vector field $\xi$ belonging to the $(k, \mu)^{\prime}$-nullity distribution and $(k, \mu)$-nullity distribution respectively, where $P$ is the Weyl projective curvature tensor. As a consequence of the main results we obtain several corollaries.
\end{abstract}

\section{Introduction}

In the present time the study of nullity distributions has become very interesting topic in Differential Geometry. Gray [7] and Tanno [12] introduced the notion of $k$-nullity distribution $(k \in \mathbb{R})$ in the study of Riemannian manifolds $(M, g)$, which is defined for any $p \in M$ and $k \in \mathbb{R}$ as follows:

$$
N_{p}(k)=\left\{Z \in T_{p} M: R(X, Y) Z=k[g(Y, Z) X-g(X, Z) Y]\right\},
$$

for any $X, Y \in T_{p} M$, where $T_{p} M$ denotes the tangent vector space of $M$ at any point $p \in M$ and $R$ denotes the Riemannian curvature tensor of type $(1,3)$.

Next Blair, Koufogiorgos and Papantoniou [3] introduced the $(k, \mu)$-nullity distribution which is a generalized notion of the $k$-nullity distribution on a contact metric manifold $\left(M^{2 n+1}, \phi, \xi, \eta, g\right)$ and defined for any $p \in M^{2 n+1}$ and $k, \mu \in \mathbb{R}$ as follows:

$$
\begin{aligned}
N_{p}(k, \mu)=\left\{Z \in T_{p} M^{2 n+1}: R(X, Y) Z=\right. & k[g(Y, Z) X-g(X, Z) Y] \\
& +\mu[g(Y, Z) h X-g(X, Z) h Y]\},
\end{aligned}
$$

where $h=\frac{1}{2} £_{\xi} \phi$ and $£$ denotes the Lie differentiation.

In [5], Dileo and Pastore introduced the notion of $(k, \mu)^{\prime}$-nullity distribution, another generalized notion of the $k$-nullity distribution, on an almost Kenmotsu manifold $\left(M^{2 n+1}\right.$, $\phi, \xi, \eta, g)$, which is defined for any $p \in M^{2 n+1}$ and $k, \mu \in \mathbb{R}$ as follows:

$$
N_{p}(k, \mu)^{\prime}=\left\{Z \in T_{p} M^{2 n+1}: R(X, Y) Z=k[g(Y, Z) X-g(X, Z) Y]\right.
$$

Received September 4, 2016, accepted Febuery 24, 2017.

2010 Mathematics Subject Classification. 53C25, 53C35.

Key words and phrases. Almost Kenmotsu manifolds, nullity distribution, Weyl projective curvature tensor, Einstein manifold.

Corresponding author: Uday Chand De. 


$$
\left.+\mu\left[g(Y, Z) h^{\prime} X-g(X, Z) h^{\prime} Y\right]\right\}
$$

where $h^{\prime}=h \circ \phi$.

Also, Kenmotsu [9] introduced a new type of almost contact metric manifolds named Kenmotsu manifolds nowadays. A differentiable $(2 n+1)$-dimensional manifold $M$ is said to have a $(\phi, \xi, \eta)$-structure or an almost contact structure, if it admits a $(1,1)$ tensor field $\phi$, a characteristic vector field $\xi$ and a 1-form $\eta$ satisfying $[1,2]$

$$
\phi^{2}=-I+\eta \otimes \xi, \eta(\xi)=1,
$$

where $I$ denote the identity endomorphism. Here we include also $\phi \xi=0$ and $\eta \circ \phi=0$; both can be derived from (1.4).

If a manifold $M$ with a $(\phi, \xi, \eta)$-structure admits a Riemannian metric $g$ such that

$$
g(\phi X, \phi Y)=g(X, Y)-\eta(X) \eta(Y)
$$

for any vector fields $X$ and $Y$ of $T_{p} M^{2 n+1}$, then $M$ is said to have an almost contact metric structure $(\phi, \xi, \eta, g)$. The fundamental 2 -form $\Phi$ is defined by $\Phi(X, Y)=g(X, \phi Y)$ for any vector fields $X, Y$ of $T_{p} M^{2 n+1}$. The condition for an almost contact metric manifold being normal is equivalent to vanishing of the $(1,2)$-type torsion tensor $N_{\phi}$, defined by $N_{\phi}=[\phi, \phi]+2 d \eta \otimes \xi$, where $[\phi, \phi]$ is the Nijenhuis torsion of $\phi[1]$. A normal almost Kenmotsu manifold is a Kenmotsu manifold such that $d \eta=0$ and $d \Phi=2 \eta \wedge \Phi$. Also Kenmotsu manifolds can be characterized by $\left(\nabla_{X} \phi\right) Y=g(\phi X, Y) \xi-\eta(Y) \phi X$ for any vector fields $X, Y$. It is well known [9] that a Kenmotsu manifold $M^{2 n+1}$ is locally a warped product $I \times_{f} N^{2 n}$, where $N^{2 n}$ is a Kähler manifold, $I$ is an open interval with coordinate $t$ and the warping function $f$, defined by $f=c e^{t}$ for some positive constant $c$. Let us denote the distribution orthogonal to $\xi$ by $\mathscr{D}$ and defined by $\mathscr{D}=\operatorname{Ker}(\eta)=\operatorname{Im}(\phi)$. In an almost Kenmotsu manifold, since $\eta$ is closed, $\mathscr{D}$ is an integrable distribution.

A Riemannian manifold $\left(M^{2 n+1}, g\right)$ is called locally symmetric if its curvature tensor $R$ is parallel, that is, $\nabla R=0$, where $\nabla$ is the Levi-Civita connection. The notion of semisymmetric manifold, a proper generalization of locally symmetric manifold, is defined by $R(X, Y) \cdot R=0$, where $R(X, Y)$ is considered as a field of linear operators, acting on $R$. A complete intrinsic classification of these manifolds was given by Szabó in [11]. In a recent paper [8] Jun, De and Pathak studied Weyl semisymmetric Kenmotsu manifolds.

Let $M$ be a $(2 n+1)$-dimensional Riemannian manifold. If there exists a one-to-one correspondence between each coordinate neighborhood of $M$ and a domain in Euclidean space such that any geodesic of the Riemannian manifold corresponds to a straight line in the Euclidean space, then $M$ is said to be locally projectively flat. For $n \geq 1, M$ is locally projectively 
flat if and only if the well-known Weyl projective curvature tensor $P$ vanishes. Here $P$ is defined by [10]

$$
P(X, Y) Z=R(X, Y) Z-\frac{1}{2 n}[S(Y, Z) X-S(X, Z) Y],
$$

for all $X, Y, Z \in T_{p} M$, where $R$ is the curvature tensor and $S$ is the Ricci tensor of type $(0,2)$ of $M$. In fact, $M$ is Weyl projectively flat if and only if the manifold is of constant curvature [17]. Thus the Weyl projective curvature tensor is the measure of the failure of a Riemannian manifold to be of constant curvature. A Riemannian manifold is said to be Weyl projective semisymmetric if the curvature tensor $P$ satisfies $R(X, Y) \cdot P=0$.

In [4], Dileo and Pastore studied locally symmetric almost Kenmotsu manifolds. We refer the reader to ([4],[5],[6]) for more related results on $(k, \mu)^{\prime}$-nullity distribution and $(k, \mu)$ nullity distribution on almost Kenmotsu manifolds. In recent papers ([13],[14],[15],[16]) Wang and Liu studied almost Kenmotsu manifolds with nullity distributions. In [14], Wang and Liu studied $\xi$-Riemannian semisymmetric almost Kenmotsu manifolds with $\xi$ belonging to the $(k, \mu)^{\prime}$-nullity distribution and $(k, \mu)$-nullity distribution.

Motivated by the above studies we study Weyl projective semisymmetric $(R \cdot P=0)$ and the curvature condition $P \cdot S=0$ in an almost Kenmotsu manifolds with nullity distributions.

The paper is organized as follows:

Section 2 focuses on almost Kenmotsu manifolds with $\xi$ belonging to the $(k, \mu)^{\prime}$-nullity distribution and $\xi$ belonging to the $(k, \mu)$-nullity distribution. In sections 3 and 4 we study Weyl projective semisymmetric almost Kenmotsu manifolds and almost Kenmotsu manifolds satisfying the curvature condition $P \cdot S=0$ with characteristic vector field $\xi$ belonging to the $(k, \mu)^{\prime}$-nullity distribution and $(k, \mu)$-nullity distribution respectively. As a consequence of the main results we obtain several corollaries.

\section{Almost Kenmotsu manifolds}

Let $M^{2 n+1}$ be an almost Kenmotsu manifold. We denote by $h=\frac{1}{2} £_{\xi} \phi$ and $l=R(\cdot, \xi) \xi$ on $M^{2 n+1}$. The tensor fields $l$ and $h$ are symmetric operators and satisfy the following relations [4]

$$
h \xi=0, l \xi=0, \operatorname{tr}(h)=0, \operatorname{tr}(h \phi)=0, h \phi+\phi h=0 .
$$

Moreover, we have the following results $[4,5]$

$$
\begin{aligned}
\nabla_{X} \xi & =-\phi^{2} X-\phi h X\left(\Rightarrow \nabla_{\xi} \xi=0\right), \\
\phi l \phi-l & =2\left(h^{2}-\phi^{2}\right), \\
R(X, Y) \xi & =\eta(X)(Y-\phi h Y)-\eta(Y)(X-\phi h X)+\left(\nabla_{Y} \phi h\right) X-\left(\nabla_{X} \phi h\right) Y,
\end{aligned}
$$


for any vector fields $X, Y$. The $(1,1)$-type symmetric tensor field $h^{\prime}=h \circ \phi$ is anticommuting with $\phi$ and $h^{\prime} \xi=0$. Also it is clear that

$$
h=0 \Leftrightarrow h^{\prime}=0, h^{\prime 2}=(k+1) \phi^{2}\left(\Leftrightarrow h^{2}=(k+1) \phi^{2}\right),
$$

which holds on $(k, \mu)^{\prime}$-almost Kenmotsu manifold.

\section{3. $\xi$ belongs to the $(k, \mu)^{\prime}$-nullity distribution}

This section is devoted to study of almost Kenmotsu manifolds with $\xi$ belonging to the $(k, \mu)^{\prime}$-nullity distribution. Let $X \in \mathscr{D}$ be the eigen vector of $h^{\prime}$ corresponding to the eigen value $\lambda$. Then from (2.5) it is clear that $\lambda^{2}=-(k+1)$, a constant. Therefore $k \leq-1$ and $\lambda=$ $\pm \sqrt{-k-1}$. We denote by $[\lambda]^{\prime}$ and $[-\lambda]^{\prime}$ the corresponding eigenspaces related to the non-zero eigen value $\lambda$ and $-\lambda$ of $h^{\prime}$, respectively. Before presenting our main theorems we recall some results:

Lemma 3.1 (Prop. 4.1 and Prop. 4.3 of [5]). Let $\left(M^{2 n+1}, \phi, \xi, \eta, g\right)$ be an almost Kenmotsu manifold such that $\xi$ belongs to the $(k, \mu)^{\prime}$-nullity distribution and $h^{\prime} \neq 0$. Then $k<-1, \mu=-2$ and Spec $\left(h^{\prime}\right)=\{0, \lambda,-\lambda\}$, with 0 as simple eigen value and $\lambda=\sqrt{-k-1}$. The distributions $[\xi] \oplus$ $[\lambda]^{\prime}$ and $[\xi] \oplus[-\lambda]^{\prime}$ are integrable with totally geodesic leaves. The distributions $[\lambda]^{\prime}$ and $[-\lambda]^{\prime}$ are integrable with totally umbilical leaves. Furthermore, the sectional curvature are given as following:

(a) $K(X, \xi)=k-2 \lambda$ if $X \in[\lambda]^{\prime}$ and

$$
K(X, \xi)=k+2 \lambda \text { if } X \in[-\lambda]^{\prime},
$$

(b) $K(X, Y)=k-2 \lambda$ if $X, Y \in[\lambda]^{\prime}$;

$K(X, Y)=k+2 \lambda$ if $X, Y \in[-\lambda]^{\prime}$ and $K(X, Y)=-(k+2)$ if $X \in[\lambda]^{\prime}, Y \in[-\lambda]^{\prime}$,

(c) $M^{2 n+1}$ has constant negative scalar curvature $r=2 n(k-2 n)$.

Lemma 3.2 (Lemma 3 of [15]). Let $\left(M^{2 n+1}, \phi, \xi, \eta, g\right)$ be an almost Kenmotsu manifold with $\xi$ belonging to the $(k, \mu)^{\prime}$-nullity distribution and $h^{\prime} \neq 0$. If $n>1$, then the Ricci operator $Q$ of $M^{2 n+1}$ is given by

$$
Q=-2 n i d+2 n(k+1) \eta \otimes \xi-2 n h^{\prime} .
$$

Moreover, the scalar curvature of $M^{2 n+1}$ is $2 n(k-2 n)$.

Lemma 3.3 (Proposition 4.2 of [5]). Let $\left(M^{2 n+1}, \phi, \xi, \eta, g\right)$ be an almost Kenmotsu manifold such that $h^{\prime} \neq 0$ and $\xi$ belongs to the $(k,-2)^{\prime}$-nullity distribution. Then for any $X_{\lambda}, Y_{\lambda}, Z_{\lambda} \in[\lambda]^{\prime}$ and $X_{-\lambda}, Y_{-\lambda}, Z_{-\lambda} \in[-\lambda]^{\prime}$, the Riemannian curvature tensor satisfies:

$$
R\left(X_{\lambda}, Y_{\lambda}\right) Z_{-\lambda}=0,
$$




$$
\begin{aligned}
R\left(X_{-\lambda}, Y_{-\lambda}\right) Z_{\lambda} & =0, \\
R\left(X_{\lambda}, Y_{-\lambda}\right) Z_{\lambda} & =(k+2) g\left(X_{\lambda}, Z_{\lambda}\right) Y_{-\lambda}, \\
R\left(X_{\lambda}, Y_{-\lambda}\right) Z_{-\lambda} & =-(k+2) g\left(Y_{-\lambda}, Z_{-\lambda}\right) X_{\lambda}, \\
R\left(X_{\lambda}, Y_{\lambda}\right) Z_{\lambda} & =(k-2 \lambda)\left[g\left(Y_{\lambda}, Z_{\lambda}\right) X_{\lambda}-g\left(X_{\lambda}, Z_{\lambda}\right) Y_{\lambda}\right], \\
R\left(X_{-\lambda}, Y_{-\lambda}\right) Z_{-\lambda} & =(k+2 \lambda)\left[g\left(Y_{-\lambda}, Z_{-\lambda}\right) X_{-\lambda}-g\left(X_{-\lambda}, Z_{-\lambda}\right) Y_{-\lambda}\right] .
\end{aligned}
$$

From (1.3) we have

$$
R(X, Y) \xi=k[\eta(Y) X-\eta(X) Y]+\mu\left[\eta(Y) h^{\prime} X-\eta(X) h^{\prime} Y\right]
$$

where $k, \mu \in \mathbb{R}$. Also we get from (3.2)

$$
R(\xi, X) Y=k[g(X, Y) \xi-\eta(Y) X]+\mu\left[g\left(h^{\prime} X, Y\right) \xi-\eta(Y) h^{\prime} X\right] .
$$

Contracting $Y$ in (3.2) we have

$$
S(X, \xi)=2 n k \eta(X) .
$$

By applying the above results and Lemma 3.2 we obtain from (1.5)

$$
P(\xi, Y) Z=(k+1) g(Y, Z) \xi-g\left(h^{\prime} Y, Z\right) \xi+2 \eta(Z) h^{\prime} Y-(k+1) \eta(Y) \eta(Z) \xi
$$

for all vector fields $Y, Z$ on $M$.

Using the above results we can present our main theorem as follows:

Theorem 3.1. Let $\left(M^{2 n+1}, \phi, \xi, \eta, g\right)(n>1)$ be an almost Kenmotsu manifold with $\xi$ belonging to the $(k, \mu)^{\prime}$-nullity distribution and $h^{\prime} \neq 0$. If the manifold $M^{2 n+1}$ is Weyl projective semisymmetric then the manifold is locally isometric to the Riemannian product of an $(n+1)$ dimensional manifold of constant sectional curvature -4 and a flat $n$-dimensional manifold.

Proof. We suppose that the manifold $M^{2 n+1}$ is Weyl projective semisymmetric, that is, $R \cdot P=$ 0 . Then $(R(X, Y) \cdot P)(U, V) W=0$ for all vector fields $X, Y, U, V, W$, which implies

$$
R(X, Y) P(U, V) W-P(R(X, Y) U, V) W-P(U, R(X, Y) V) W-P(U, V) R(X, Y) W=0 .
$$

Setting $X=U=\xi$ in (3.6) we have,

$$
R(\xi, Y) P(\xi, V) W-P(R(\xi, Y) \xi, V) W-P(\xi, R(\xi, Y) V) W-P(\xi, V) R(\xi, Y) W=0 .
$$

Making use of (3.3) and (3.5) we get

$$
\begin{aligned}
R(\xi, Y) P(\xi, V) W= & k[g(Y, P(\xi, V) W) \xi-\eta(P(\xi, V) W) Y] \\
& -2\left[g\left(h^{\prime} Y, P(\xi, V) W\right) \xi-\eta(P(\xi, V) W) h^{\prime} Y\right]
\end{aligned}
$$




$$
\begin{aligned}
= & k\left\{(k+1) g(V, W) \eta(Y) \xi-g\left(h^{\prime} V, W\right) \eta(Y) \xi+2 \eta(W) g\left(Y, h^{\prime} V\right) \xi\right. \\
& -(k+1) \eta(V) \eta(W) \eta(Y) \xi-(k+1) g(V, W) Y+g\left(h^{\prime} V, W\right) Y \\
& +(k+1) \eta(V) \eta(W) Y\}-2\left\{2 \eta(W) g\left(h^{\prime} Y, h^{\prime} V\right) \xi\right. \\
& \left.-(k+1) g(V, W) h^{\prime} Y+g\left(h^{\prime} V, W\right) h^{\prime} Y+(k+1) \eta(V) \eta(W) h^{\prime} Y\right\}
\end{aligned}
$$

for any vector fields $Y, V, W$ on $M^{2 n+1}$.

With the help of (3.3) and (3.5) we obtain

$$
\begin{aligned}
P(R(\xi, Y) \xi, V) W= & k \eta(Y) P(\xi, V) W-k P(Y, V) W+2 P\left(h^{\prime} Y, V\right) W \\
= & k(k+1) g(V, W) \eta(Y) \xi-k g\left(h^{\prime} V, W\right) \eta(Y) \xi+2 k \eta(Y) \eta(W) h^{\prime} V \\
& -k(k+1) \eta(Y) \eta(V) \eta(W) \xi-k P(Y, V) W+2 P\left(h^{\prime} Y, V\right) W
\end{aligned}
$$

for any vector fields $Y, V, W$ on $M^{2 n+1}$.

Similarly, it follows from (3.3) and (3.5) that

$$
\begin{aligned}
P(\xi, R(\xi, Y) V) W= & -k \eta(V) P(\xi, Y) W+2 \eta(V) P\left(\xi, h^{\prime} Y\right) W \\
= & -k(k+1) g(Y, W) \eta(V) \xi+k g\left(h^{\prime} Y, W\right) \eta(V) \xi-2 k \eta(V) \eta(W) h^{\prime} Y \\
& +2(k+1) g\left(h^{\prime} Y, W\right) \eta(V) \xi+2(k+1) g(Y, W) \eta(V) \xi \\
& -4(k+1) \eta(V) \eta(W) Y+(k+1)(k+2) \eta(Y) \eta(V) \eta(W) \xi
\end{aligned}
$$

for any vector fields $Y, V, W$ on $M^{2 n+1}$.

Again using (3.3) and (3.5) we obtain

$$
\begin{aligned}
P(\xi, V) R(\xi, Y) W= & k(k+1) g(Y, W) \eta(V) \xi-k(k+1) g(Y, V) \eta(W) \xi \\
& +2(k+1) g\left(h^{\prime} Y, V\right) \eta(W) \xi+k g\left(h^{\prime} V, Y\right) \eta(W) \xi-2 g\left(h^{\prime} V, h^{\prime} Y\right) \eta(W) \xi \\
& +2 k g(Y, W) h^{\prime} V-2 k \eta(Y) \eta(W) h^{\prime} V-4 g\left(h^{\prime} Y, W\right) h^{\prime} V \\
& -k(k+1) g(Y, W) \eta(V) \xi+k(k+1) \eta(Y) \eta(W) \eta(V) \xi
\end{aligned}
$$

for any vector fields $Y, V, W$ on $M^{2 n+1}$.

Finally, using (3.8)-(3.11) we have from (3.7)

$$
\begin{aligned}
& k P(Y, V) W-2 P\left(h^{\prime} Y, V\right) W+k g\left(h^{\prime} V, Y\right) \eta(W) \xi+2(k+1) g(V, W) h^{\prime} Y \\
& -k(k+1) g(V, W) Y+k g\left(h^{\prime} V, W\right) Y+\left(k^{2}+5 k+4\right) \eta(V) \eta(W) Y \\
& -2 g\left(h^{\prime} Y, h^{\prime} V\right) \eta(W) \xi-2(k+1)^{2} \eta(Y) \eta(V) \eta(W) \xi \\
& -2 g\left(h^{\prime} V, W\right) h^{\prime} Y-2 \eta(V) \eta(W) h^{\prime} Y+\left(k^{2}-k-2\right) g(Y, W) \eta(V) \xi \\
& -(3 k+2) g\left(h^{\prime} Y, W\right) \eta(V) \xi+k(k+1) g(Y, V) \eta(W) \xi
\end{aligned}
$$




$$
-2(k+1) g\left(h^{\prime} Y, V\right) \eta(W) \xi-2 k g(Y, W) h^{\prime} V+4 g\left(h^{\prime} Y, W\right) h^{\prime} V=0
$$

for any vector fields $Y, V, W$ on $M^{2 n+1}$. Letting $Y, W \in[\lambda]^{\prime}$ and $V \in[-\lambda]^{\prime}$ and applying Lemma 3.3 we have

$$
P(Y, V) W=(k+1-\lambda) g(Y, W) V \text { and } P\left(h^{\prime} Y, V\right) W=(\lambda+1)(k+1) g(Y, W) V .
$$

By using (3.13) and noticing $Y, W \in[\lambda]^{\prime}$ and $V \in[-\lambda]^{\prime}$ it follows from (3.12) that

$$
\left[k(k+1-\lambda)-2(\lambda+1)(k+1)+2 \lambda k-4 \lambda^{2}\right] g(Y, W) V=0 .
$$

Using the relationship $\lambda= \pm \sqrt{-k-1}$ in (3.14) we get

$$
\lambda(\lambda+1)^{2}(\lambda-1)=0
$$

If $\lambda=0$, then $k=-1$ and consequently from (2.5) $h^{\prime}=0$, which contradicts our hypothesis $h^{\prime} \neq 0$. Then it follows from (3.15) that $\lambda^{2}=1$ and hence $k=-2$. Without losing generality we may choose $\lambda=1$. Then we can write from Lemma 3.3

$$
\begin{aligned}
R\left(X_{\lambda}, Y_{\lambda}\right) Z_{\lambda} & =-4\left[g\left(Y_{\lambda}, Z_{\lambda}\right) X_{\lambda}-g\left(X_{\lambda}, Z_{\lambda}\right) Y_{\lambda}\right], \\
R\left(X_{-\lambda}, Y_{-\lambda}\right) Z_{-\lambda} & =0
\end{aligned}
$$

for any $X_{\lambda}, Y_{\lambda}, Z_{\lambda} \in[\lambda]^{\prime}$ and $X_{-\lambda}, Y_{-\lambda}, Z_{-\lambda} \in[-\lambda]^{\prime}$. Also it follows from Lemma 3.1 that $K(X, \xi)$ $=-4$ for any $X \in[\lambda]^{\prime}$ and $K(X, \xi)=0$ for any $X \in[-\lambda]^{\prime}$. Again from Lemma 3.1 we see that $K(X, Y)=-4$ for any $X, Y \in[\lambda]^{\prime} ; K(X, Y)=0$ for any $X, Y \in[-\lambda]^{\prime}$ and $K(X, Y)=0$ for any $X \in[\lambda]^{\prime}, Y \in[-\lambda]^{\prime}$. As is shown in [5] that the distribution $[\xi] \oplus[\lambda]^{\prime}$ is integrable with totally geodesic leaves and the distribution $[-\lambda]^{\prime}$ is integrable with totally umbilical leaves by $H=-(1-\lambda) \xi$, where $H$ is the mean curvature vector field for the leaves of $[-\lambda]^{\prime}$ immersed in $M^{2 n+1}$. Here $\lambda=1$, then two orthogonal distributions $[\xi] \oplus[\lambda]^{\prime}$ and $[-\lambda]^{\prime}$ are both integrable with totally geodesic leaves immersed in $M^{2 n+1}$. Then we can say that $M^{2 n+1}$ is locally isometric to $\mathbb{H}^{n+1}(-4) \times \mathbb{R}^{n}$. This completes the proof of our theorem.

Since $R \cdot R=0$ implies $R \cdot P=0$, we have the following:

Corollary 3.1. A semisymmetric almost Kenmotsu manifold $M^{2 n+1}(n>1)$ with $\xi$ belonging to the $(k, \mu)^{\prime}$-nullity distribution and $h^{\prime} \neq 0$ is locally isometric to the Riemannian product of an $(n+1)$-dimensional manifold of constant sectional curvature -4 and a flat $n$-dimensional manifold.

The above corollary have been proved by Wang and Liu [14]. 
Next we consider an almost Kenmotsu manifold with $\xi$ belonging to the $(k, \mu)^{\prime}$-nullity distribution and $h^{\prime} \neq 0$ satisfying the curvature condition $P \cdot S=0$. Then $(P(X, Y) \cdot S)(U, V)=0$ for all vector fields $X, Y, U, V$, which implies

$$
S(P(X, Y) U, V)+S(U, P(X, Y) V)=0
$$

for any vector fields $X, Y, U, V$ on $M^{2 n+1}$.

Putting $X=U=\xi$ in (3.16) we have,

$$
S(P(\xi, Y) \xi, V)+S(\xi, P(\xi, Y) V)=0 .
$$

Making use of (3.4) and (3.5) the above equation implies

$$
S\left(h^{\prime} Y, V\right)+n k(k+1) g(Y, V)-n k g\left(h^{\prime} Y, V\right)-n k(k+1) \eta(Y) \eta(V)=0
$$

for any vector fields $Y, V$ on $M^{2 n+1}$.

Substituting $Y=h^{\prime} Y$ in (3.18) and using (2.5) we obtain

$$
(k+1)\left\{-S(Y, V)+n k \eta(Y) \eta(V)+n k g\left(h^{\prime} Y, V\right)+n k g(Y, V)\right\}=0
$$

for any vector fields $Y, V$ on $M^{2 n+1}$.

Again from Lemma 3.2 we have

$$
S(Y, V)=-2 n g(Y, V)+2 n(k+1) \eta(Y) \eta(V)-2 n g\left(h^{\prime} Y, V\right)
$$

for any vector fields $Y, V$ on $M^{2 n+1}$.

Making use of (3.20) we obtain from (3.19)

$$
(k+1)(k+2)\left\{g(Y, V)+g\left(h^{\prime} Y, V\right)-\eta(Y) \eta(V)\right\}=0 .
$$

Letting $Y, V \in[\lambda]^{\prime}$ in (3.21) implies that

$$
(k+1)(k+2)(1+\lambda) g(Y, V)=0 .
$$

Using the relation $\lambda= \pm \sqrt{-k-1}$ in (3.22) we have

$$
\lambda^{2}(\lambda+1)^{2}(\lambda-1)=0
$$

Suppose $\lambda=0$, then $k=-1$ and hence it follows from (2.5) that $h^{\prime}=0$, which contradicts our hypothesis $h^{\prime} \neq 0$. Then from (3.23) we have $\lambda^{2}=1$ and hence $k=-2$. Without losing the generality, we may choose $\lambda=1$. Then we can write from Lemma 3.3

$$
R\left(X_{\lambda}, Y_{\lambda}\right) Z_{\lambda}=-4\left[g\left(Y_{\lambda}, Z_{\lambda}\right) X_{\lambda}-g\left(X_{\lambda}, Z_{\lambda}\right) Y_{\lambda}\right]
$$




$$
R\left(X_{-\lambda}, Y_{-\lambda}\right) Z_{-\lambda}=0
$$

for any $X_{\lambda}, Y_{\lambda}, Z_{\lambda} \in[\lambda]^{\prime}$ and $X_{-\lambda}, Y_{-\lambda}, Z_{-\lambda} \in[-\lambda]^{\prime}$. Also it follows from Lemma 3.1 that $K(X, \xi)$ $=-4$ for any $X \in[\lambda]^{\prime}$ and $K(X, \xi)=0$ for any $X \in[-\lambda]^{\prime}$. Again from Lemma 3.1 we see that $K(X, Y)=-4$ for any $X, Y \in[\lambda]^{\prime} ; K(X, Y)=0$ for any $X, Y \in[-\lambda]^{\prime}$ and $K(X, Y)=0$ for any $X \in[\lambda]^{\prime}, Y \in[-\lambda]^{\prime}$. As is shown in [5] that the distribution $[\xi] \oplus[\lambda]^{\prime}$ is integrable with totally geodesic leaves and the distribution $[-\lambda]^{\prime}$ is integrable with totally umbilical leaves by $H=-(1-\lambda) \xi$, where $H$ is the mean curvature vector field for the leaves of $[-\lambda]^{\prime}$ immersed in $M^{2 n+1}$. Here $\lambda=1$, then two orthogonal distributions $[\xi] \oplus[\lambda]^{\prime}$ and $[-\lambda]^{\prime}$ are both integrable with totally geodesic leaves immersed in $M^{2 n+1}$. Then we can say that $M^{2 n+1}$ is locally isometric to $\mathbb{H}^{n+1}(-4) \times \mathbb{R}^{n}$. By the above discussions we can state the following:

Theorem 3.2. Let $\left(M^{2 n+1}, \phi, \xi, \eta, g\right)(n>1)$ be an almost Kenmotsu manifold with $\xi$ belonging to the $(k, \mu)^{\prime}$-nullity distribution and $h^{\prime} \neq 0$. If the manifold satisfies the curvature condition $P \cdot S=0$, then the manifold is locally isometric to the Riemannian product of an $(n+1)$ dimensional manifold of constant sectional curvature -4 and a flat $n$-dimensional manifold.

\section{4. $\xi$ belongs to the $(k, \mu)$-nullity distribution}

In this section we deal with almost Kenmotsu manifolds of which $\xi$ belonging to the $(k, \mu)$-nullity distribution.

From (1.2) we obtain

$$
R(X, Y) \xi=k[\eta(Y) X-\eta(X) Y]+\mu[\eta(Y) h X-\eta(X) h Y]
$$

where $k, \mu \in \mathbb{R}$. Before proving our main results in this section we state the following:

Lemma 4.1 (Theorem 4.1 of [5]). Let $M$ be an almost Kenmotsu manifold of dimension $2 n+1$. Suppose that the characteristic vector field $\xi$ belonging to the $(k, \mu)$-nullity distribution. Then $k=-1, h=0$ and $M$ is locally a warped product of an open interval and an almost Kähler manifold.

In view of Lemma 4.1 it follows from (4.1) that

$$
\begin{aligned}
R(X, Y) \xi & =\eta(X) Y-\eta(Y) X, \\
R(\xi, X) Y & =-g(X, Y) \xi+\eta(Y) X, \\
S(X, \xi) & =-2 n \eta(X)
\end{aligned}
$$

for any vector fields $X, Y$ on $M^{2 n+1}$. 
Applying (4.3) and (4.4) in (1.5) we have the following

$$
P(\xi, Y) Z=-g(Y, Z) \xi-\frac{1}{2 n} S(Y, Z) \xi
$$

for any vector fields $Y, Z$ on $M^{2 n+1}$. We can state our main theorem as follows:

Theorem 4.1. An almost Kenmotsu manifold $\left(M^{2 n+1}, \phi, \xi, \eta, g\right)$ with $\xi$ belonging to the $(k, \mu)$ nullity distribution is Weyl projective semisymmetric if and only if the manifold is of constant curvature-1.

Proof. Let $M^{2 n+1}$ be a Weyl projective semisymmetric almost Kenmotsu manifold with $\xi$ belonging to the $(k, \mu)$-nullity distribution. Therefore $(R(X, Y) \cdot P)(U, V) W=0$ for all vector fields $X, Y, U, V, W$, which implies

$$
R(X, Y) P(U, V) W-P(R(X, Y) U, V) W-P(U, R(X, Y) V) W-P(U, V) R(X, Y) W=0 .
$$

Substituting $X=U=\xi$ in (4.6) we obtain

$$
R(\xi, Y) P(\xi, V) W-P(R(\xi, Y) \xi, V) W-P(\xi, R(\xi, Y) V) W-P(\xi, V) R(\xi, Y) W=0 .
$$

Making use of (4.3) and (4.5) we have

$$
\begin{aligned}
R(\xi, Y) P(\xi, V) W= & g(V, W) \eta(Y) \xi+\frac{1}{2 n} S(V, W) \eta(Y) \xi \\
& -g(V, W) Y-\frac{1}{2 n} S(V, W) Y
\end{aligned}
$$

for any vector field $Y, V, W$ on $M^{2 n+1}$.

Similarly using (4.3) and (4.5) we obtain

$$
P(R(\xi, Y) \xi, V) W=P(Y, V) W+g(V, W) \eta(Y) \xi+\frac{1}{2 n} S(V, W) \eta(Y) \xi
$$

for any vector field $Y, V, W$ on $M^{2 n+1}$.

Again, it follows from (4.3) and (4.5) that

$$
P(\xi, R(\xi, Y) V) W=-g(Y, W) \eta(V) \xi-\frac{1}{2 n} S(Y, W) \eta(V) \xi
$$

for any vector field $Y, V, W$ on $M^{2 n+1}$.

Finally, using (4.3) and (4.5) we have

$$
P(\xi, V) R(\xi, Y) W=-g(V, Y) \eta(W) \xi-\frac{1}{2 n} S(V, Y) \eta(W) \xi
$$

for any vector field $Y, V, W$ on $M^{2 n+1}$. 
Substituting (4.8)-(4.11) into (4.7) gives

$$
\begin{aligned}
P(Y, V) W= & -g(V, W) Y-\frac{1}{2 n} S(V, W) Y+g(Y, W) \eta(V) \xi+\frac{1}{2 n} S(Y, W) \eta(V) \xi \\
& +g(V, Y) \eta(W) \xi+\frac{1}{2 n} S(V, Y) \eta(W) \xi
\end{aligned}
$$

for any vector field $Y, V, W$ on $M^{2 n+1}$.

In view of (1.5) and (4.12) we obtain

$$
\begin{aligned}
R(Y, V) W= & -g(V, W) Y+g(Y, W) \eta(V) \xi+\frac{1}{2 n} S(Y, W) \eta(V) \xi \\
& +g(V, Y) \eta(W) \xi+\frac{1}{2 n} S(V, Y) \eta(W) \xi-\frac{1}{2 n} S(Y, W) V .
\end{aligned}
$$

Contracting $Y$ in (4.13) it follows that

$$
S(V, W)=-2 n g(V, W)
$$

for any vector field $V, W$ on $M^{2 n+1}$.

Taking account of (4.14) we have from (4.13)

$$
R(Y, V) W=-[g(V, W) Y-g(Y, W) V]
$$

that is, the manifold is of constant curvature -1 .

Conversely, if the manifold is of constant curvature -1 then obviously Weyl projective semisymmetry follows. This completes the proof.

Since $R \cdot R=0$ implies $R \cdot P=0$, we have the following:

Corollary 4.1. An almost Kenmotsu manifold $\left(M^{2 n+1}, \phi, \xi, \eta, g\right)$ with $\xi$ belonging to the $(k, \mu)$ nullity distribution is semisymmetric if and only if the manifold is of constant curvature -1 .

The above corollary have been proved by Wang and Liu [14].

Let $M^{2 n+1}$ be an almost Kenmotsu manifold with $\xi$ belonging to the $(k, \mu)$-nullity distribution satisfying the curvature condition $P \cdot S=0$. Then $(P(X, Y) \cdot S)(U, V)=0$ for all vector fields $X, Y, U, V$, which implies

$$
S(P(X, Y) U, V)+S(U, P(X, Y) V)=0
$$

for any vector fields $X, Y, U, V$ on $M^{2 n+1}$.

Setting $X=U=\xi$ in (4.16) we have,

$$
S(P(\xi, Y) \xi, V)+S(\xi, P(\xi, Y) V)=0 .
$$


Using (4.4) and (4.5) we obtain from (4.17)

$$
\eta(P(\xi, Y) V)=0
$$

In view of (4.5) and (4.18) it follows that

$$
S(Y, V)=-2 n g(Y, V),
$$

which implies that the manifold is an Einstein manifold.

Conversely, let the manifold be an Einstein manifold of the form (4.19). Then it is obvious that $P \cdot S=0$. This leads to the following:

Theorem 4.2. An almost Kenmotsu manifold $M^{2 n+1}$ with $\xi$ belonging to the $(k, \mu)$-nullity distribution satisfies the curvature condition $P \cdot S=0$ if and only if the manifold is an Einstein one.

\section{References}

[1] D. E. Blair, Contact manifolds in Riemannian geometry, Lecture Notes on Mathematics, Springer, Berlin, 509, 1976.

[2] D. E. Blair, Riemannian geometry on contact and symplectic manifolds, Progr. Math., 203, Birkhäuser, 2010.

[3] D. E. Blair, T. Koufogiorgos and B. J. Papantoniou, Contact metric manifolds satisfying a nullity condition, Israel. J. Math. 91 (1995), 189-214.

[4] G. Dileo and A. M. Pastore, Almost Kenmotsu manifolds and local symmetry, Bull. Belg. Math. Soc. Simon Stevin, 14(2007), 343-354.

[5] G. Dileo and A. M. Pastore, Almost Kenmotsu manifolds and nullity distributions, J. Geom. 93(2009), 46-61.

[6] G. Dileo and A. M. Pastore, Almost Kenmotsu manifolds with a condition of $\eta$-parallelism, Differential Geom. Appl. 27(2009), 671-679.

[7] A. Gray, Spaces of constancy of curvature operators, Proc. Amer. Math. Soc., 17(1966), 897-902.

[8] J.-B. Jun, U.C. De and G. Pathak, On Kenmotsu manifolds, J. Korean Math. Soc. 42(2005), 435-445.

[9] K. Kenmotsu, A class of almost contact Riemannian manifolds, Tohoku Math. J. 24(1972), 93-103.

[10] G. Sooś, Über die geodätischen Abbildungen von Riemannaschen Räumen auf projektiv symmetrische Riemannsche Räume, Acta. Math. Acad. Sci. Hungar., 9(1958), 359-361.

[11] Z. I. Szabó, Structure theorems on Riemannian spaces satisfying $R(X, Y) \cdot R=0$, the local version, J. Diff. Geom., 17(1982), 531-582.

[12] S. Tanno, Some differential equations on Riemannian manifolds, J. Math. Soc. Japan, 30(1978), 509-531.

[13] Y. Wang and X. Liu, Second order parallel tensors on almost Kenmotsu manifolds satisfying the nullity distributions, Filomat 28(2014), 839-847.

[14] Y. Wang and X. Liu, Riemannian semisymmetric almost Kenmotsu manifolds and nullity distributions, Ann. Polon. Math. 112(2014), 37-46.

[15] Y. Wang and X. Liu, On $\phi$-recurrent almost Kenmotsu manifolds, Kuwait J. Sci. 42(2015), 65-77.

[16] Y. Wang and X. Liu, On a type of almost Kenmotsu manifolds with harmonic curvature tensors, Bull. Belg. Math. Soc. Simon Stevin 22(2015), 15-24.

[17] K. Yano and S. Bochner, Curvature and Betti numbers, Ann. Math. Stud. 32(1953). 
Department of Pure Mathematics, University of Calcutta, 35, Ballygunge Circular Road, Kol- 700019, West Bengal, INDIA.

E-mail: uc_de@yahoo.com

Department of Mathematics, College of Natural Science, Kookmin University, Seoul 136-702, KOREA.

E-mail: jbjun@kookmin.ac.kr

Department of Pure Mathematics, University of Calcutta, 35, Ballygunge Circular Road, Kol- 700019, West Bengal, INDIA.

E-mail: krishanu.mandal013@gmail.com 